\title{
Crecimiento de Cedrela odorata e incidencia de Hypsipyla grandella en respuesta al manejo nutrimental
}

\author{
Growth of Cedrela odorata and incidence of Hypsipyla grandella \\ as affected by nutrient management
}

\author{
Celi Gloria Calixto ${ }^{\text {a*}}$, Miguel A López ${ }^{\text {a }}$, Armando Equihua ${ }^{\text {b}}$, Diego E Lira ${ }^{c}$, Víctor M Cetina ${ }^{\text {a }}$

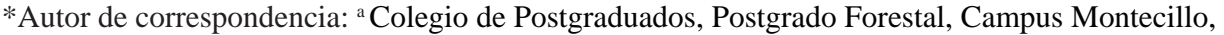 \\ km 36,5 carretera México-Texcoco, Montecillo, Estado de México, C. P. 56230, Calixto.celi@colpos.mx. \\ ${ }^{\text {b } C o l e g i o ~ d e ~ P o s t g r a d u a d o s, ~ P o s t g r a d o ~ e n ~ F i t o p a t o l o g i ́ a, ~ E s t a d o ~ d e ~ M e ́ x i c o . ~}$ \\ c Bayer, México.
}

\section{SUMMARY}

Cedrela odorata is one of the most important native forest species in México; however, plantation projects with this species have been limited because of the irruption of the meliaceae shoot borer. The search for new alternatives to either eradicate or control the pest continues being important. Hence, suitability of nutrient management through fertilization is analyzed as a method to overcome this limitation. Therefore, the effects of the application of nitrogen, phosphorus and potassium on both the growth of $C$. odorata and the incidence of $H$. grandella were evaluated in a Spanish cedar forest plantation located in Papantla Veracruz, Mexico. Periodic measurements of height and diameter growth, and the other variables related to pest incidence, were made over a year. There were positive responses on the growth with nitrogen application. A pest attack reduction was shown with potassium supply; nevertheless, these were limited because of different conditions of the plantation. Despite of this, nutrient management through fertilization shows potential as an H. grandella control method in C. odorata forest plantations.

Key words: Spanish-cedar, forest pest, forest fertilization.

\section{RESUMEN}

Cedrela odorata es una de las especies nativas de mayor importancia forestal en México; sin embargo, el desarrollo de plantaciones se ha visto limitado por la presencia del barrenador de las meliáceas. La búsqueda de alternativas para erradicar o controlar la plaga continúa siendo preponderante. En el presente, se analiza la factibilidad del manejo nutrimental a través de fertilización como método contra esta problemática. Para ello, en una plantación de cedro rojo ubicada en Papantla, Veracruz, mediante un experimento factorial se evaluaron los efectos de la aplicación de nitrógeno, fósforo y potasio, sobre el crecimiento de C. odorata e incidencia de Hypsipyla grandella. Durante un año, se realizaron evaluaciones periódicas en campo de crecimiento en altura y diámetro, y de otras variables relacionadas con la incidencia de la plaga. Existieron respuestas positivas en el crecimiento mediante la aplicación de nitrógeno y se manifestó una reducción del ataque con el suministro del potasio, pero en general estas fueron limitadas debido a diversas condiciones de la plantación. A pesar de esto, la fertilización mostró potencial como método de control de la incidencia de $H$. grandella en plantaciones de C. odorata.

Palabras clave: cedro rojo, plaga forestal, fertilización forestal.

\section{INTRODUCCIÓN}

A menudo, estadísticas nacionales evidencian que México es un país deficitario en recursos forestales. Se estima que se necesitan 20,5 millones de metros cúbicos anuales de madera en rollo para satisfacer la demanda nacional de productos forestales (Mery et al. 2001), pues solo el $42 \%$ de la demanda nacional es cubierta por la producción local. Para cubrir el déficit nacional e impulsar el desarrollo de la industria forestal del país, se han propuesto diversas estrategias. El establecimiento de plantaciones forestales, principalmente en los trópicos, ha sido una alternativa para este fin.

Se ha impulsado el uso de especies nativas en tales proyectos debido al alto valor económico, ecológico y social que varias especies poseen. Cedrela odorata L. es una de las principales, al ser una de las especies base de la industria forestal nacional de maderas preciosas. Sin embargo, el establecimiento y la productividad de plantaciones forestales con esta especie ha sido limitada, por un lado, debido a la falta de información silvícola, y, por otro, por la incidencia alta de plagas y enfermedades. 
Hypsipyla grandella Zeller (Insecta: Lepidoptera: Pyralida: Physitinae), comúnmente conocida como "barrenador de las meliaceas", es una de las principales plagas que ataca a dicha especie (Cibrián et al. 1995, Pennington y Sarukhan 2005). El problema que esta representa, básicamente se relaciona con la deformación de los tallos o con la mortalidad alta en plantaciones jóvenes cuando sus ataques son continuos (Cibrián et al. 1995, Briceño 1997).

Aunque no se ha logrado erradicar la plaga de las plantaciones, se cuenta con diversas alternativas para reducir su incidencia. Algunas son efectivas, como los métodos de control químico y biológico (Manceboa et al. 2002, Goulet et al. 2005, Lim et al. 2008, Barboza et al. 2010); sin embargo, no son económica ni ecológicamente rentables. Otras, que se basan en aspectos silvícolas, como las plantaciones mixtas, densidades de plantación e injertos con especies resistentes (Opuni-Frimpong et al. 2008, Pérez-Salicrup y Esquivel 2008, Julian et al. 2010) son más rentables económica o ecológicamente, pero tienen el inconveniente de ser técnicamente poco efectivas. De ahí que la búsqueda de nuevas alternativas ya sea para erradicar o controlar $H$. grandella sigue siendo una tarea preponderante.

Briseño (1997) reporta que la fase más susceptible de las plantaciones de C. odorata al ataque del barrenador, comprende, por el tamaño de los árboles, los primeros tres a seis años de edad. Si durante ese lapso no son atacados, es posible que la plaga ya no represente un problema para la plantación, a menos que el desempeño de los individuos sea pobre en términos de crecimiento y continúen siendo vulnerables. Entonces, un método que promueva en los árboles un crecimiento rápido y vigoroso con el cual puedan evadir rápidamente la fase de vulnerabilidad, al mismo tiempo que proporcione resistencia al ataque de la plaga, es conveniente para encarar la problemática de H. grandella. En Swietenia macrophylla King, la evidencia silvicultural aportada por Silva et al. (2014) muestra que a través de un manejo nutrimental adecuado, en términos de nutrientes y dosis aplicadas, se tienen tales atributos.

Por lo tanto, quizá esta sea una alternativa viable para reducir el ataque de $H$. grandella en C. odorata. En el presente trabajo, se prueba con dosis distintas: dosis de nitrógeno, fósforo y potasio de manera individual y combinada, si el manejo nutrimental, a través de la fertilización, es un método factible para la problemática anterior.

\section{MÉTODOS}

Descripción del área de estudio. El presente trabajo se realizó en una plantación de C. odorata, establecida en junio de 2011, en Papantla, Veracruz, México. La plantación llamada "El Cedral" está localizada en la comunidad de Arroyo Colorado municipio de Papantla, Veracruz, en las coordenadas geográficas $20^{\circ} 28^{\prime} 44^{\prime \prime} \mathrm{N}$ y $97^{\circ} 18^{\prime} 20^{\prime \prime} \mathrm{O}$. El suelo del sitio de estudio tiene las siguientes propiedades físico-químicas (cuadro 1).
Cuadro 1. Propiedades físicas y químicas del suelo del área de estudio*.

Physical and chemical properties of soil in the study area.

\begin{tabular}{lc}
\hline Características del suelo & Valor \\
\hline Nitrógeno $\left(\mathrm{mg} \mathrm{kg}^{-1}\right)$ & 30,5 \\
Fósforo $\left(\mathrm{mg} \mathrm{kg}^{-1}\right)$ & 11,3 \\
Potasio $\left(\mathrm{mg} \mathrm{kg}^{-1}\right)$ & 238,7 \\
Calcio $\left(\mathrm{mg} \mathrm{kg}^{-1}\right)$ & $7.989,0$ \\
Magnesio $\left(\mathrm{mg} \mathrm{kg}^{-1}\right)$ & 187,3 \\
Hierro $\left(\mathrm{mg} \mathrm{kg}^{-1}\right)$ & 7,3 \\
Cobre $\left(\mathrm{mg} \mathrm{kg}^{-1}\right)$ & 0,25 \\
Zinc (mg kg-1) & 0,57 \\
Manganeso (mg kg-1) & 2,24 \\
Boro (mg kg $\left.{ }^{-1}\right)$ & 1,38 \\
Arena (\%) & 34,2 \\
Limo (\%) & 39,3 \\
Arcilla (\%) & 26,5 \\
Materia orgánica (\%) & 4,1 \\
pH & 8,07 \\
Textura & Franca arcillosa \\
\hline
\end{tabular}

*Análisis realizado en el Laboratorio del Departamento de suelos de la Universidad Autónoma Chapingo.

Establecimiento del experimento. Fue con un diseño factorial $2^{3}$. Los factores fueron nitrógeno, fósforo y potasio, cada uno de ellos aplicado en dos niveles: sin y con nutrimento. La combinación de los niveles de los factores produjo ocho tratamientos (cuadro 2), de los cuales, cada uno se replicó 20 veces. La unidad experimental fue un árbol y la distribución de los tratamientos en el área experimental se realizó en forma completamente al azar.

Aplicación de nutrimentos y evaluación de las variables. El cálculo de las dosis de fertilización se hizo con base en la fórmula racional: dosis = (demanda - suministro $) /$ eficiencia, descrita por Álvarez et al. (1999). Para ello, se consideró: 1) la edad de los árboles de C. odorata; 2) un nivel estimado de eficiencia de la fertilización y 3) la composición foliar determinada a partir de un análisis químico.

Se realizaron dos aplicaciones de fertilizante: la primera aplicación se realizó el 30 de marzo de 2012 y la segunda el 06 de diciembre del mismo año. Los materiales fertilizantes fueron aplicados en la zona de goteo del árbol. Se midió la altura y el diámetro inicial de los árboles junto con la primera fertilización. Posteriormente, durante un año, se realizaron evaluaciones periódicas de estas 
Cuadro 2. Tratamientos de fertilización química aplicados a $\mathrm{Ce}$ drela odorata.

Chemical fertilization treatments applied on C. odorata.

\begin{tabular}{cccc}
\hline Tratamiento & Urea (g) & $\begin{array}{c}\text { Superfosfato } \\
\text { simple de calcio (g) }\end{array}$ & $\begin{array}{c}\text { Sulfato de } \\
\text { potasio (g) }\end{array}$ \\
\hline 1 & 0 & 0 & 0 \\
2 & 0 & 0 & 10 \\
3 & 0 & 7,5 & 0 \\
4 & 0 & 7,5 & 10 \\
5 & 5 & 0 & 0 \\
6 & 5 & 0 & 10 \\
7 & 5 & 7,5 & 0 \\
8 & 5 & 7,5 & 10 \\
\hline
\end{tabular}

mismas variables para determinar los incrementos a través del tiempo. Éstas fueron efectuadas en agosto, octubre y diciembre de 2012, y febrero y abril de 2013. La altura se determinó con una regla telescópica desde la base del tallo hasta el ápice principal, y el diámetro se midió en la base del tallo del árbol utilizando un vernier digital marca Mitutoyo. Adicionalmente, para la evaluación del daño de la plaga, bimestralmente se analizaron las siguientes variables:1) número total de brotes por árbol de $C$. odorata (se contabilizó el número de brotes totales generados en el año en curso, presentes en el período de evaluación); 2) número de brotes atacados por árbol (se examinó cada uno de los brotes del año en curso y se registró su condición de sano o atacado); 3) número de ataques por brote (para cada brote se registró el número de perforaciones presentes relacionadas con el ataque de $H$. grandella); 4) número total de ataques por árbol (esta variable resultó de integrar la variable anterior a nivel de árbol).

Por otro lado, con relación a las variables fisiológicas, se evaluó contenido de nitrógeno, fósforo y potasio. Para ello, se utilizaron los datos de biomasa y la concentración de los nutrimentos de las muestras enviadas al laboratorio. Las concentraciones de los nutrimentos se determinaron a partir de cuatro muestras compuestas de tejido vegetal (follaje) por tratamiento. El nitrógeno se determinó por método Micro-Kjeldahl, el fósforo mediante fotocolorimetría por reducción con Vanadato-Molibdato y el potasio a través de la lectura directa del digestado en un espectrómetro de absorción atómica.

Control de maleza en el sitio de estudio. Debido a la abundante presencia de malezas en la plantación, fueron requeridas operaciones de deshierbe. Inicialmente, la maleza se controló mecánicamente cada dos meses después de establecido el experimento; sin embargo, al haber árboles cortados accidentalmente, se optó por la aplicación de una mezcla de herbicidas (2-4 D amina + glifosato + indaziflam), la cual se aplicó con una bomba de aspersión el 06 de octubre de 2012.

Análisis estadísticos. Los datos de crecimiento fueron procesados mediante un análisis de covarianza utilizando como covariable el diámetro inicial de los árboles. Las variables relacionadas con incidencia de la plaga, se analizaron mediante la prueba de Kruskal-Wallis o Wilcoxon. Las variables fisiológicas se evaluaron mediante análisis de varianza factorial. En casos requeridos, las medias se compararon mediante la prueba de Tukey $(\alpha=0,05)$. El procesamiento estadístico de los datos se hizo con el paquete estadístico SAS (versión 9.0). La concentración y contenido de los nutrimentos se evaluó mediante análisis de graficas de vectores en la que se utilizaron los valores de concentración y peso seco de tejido vegetal.

\section{RESULTADOS}

Nutrimentos individuales aplicados y sus interacciones sobre el crecimiento de C. odorata. De acuerdo con el análisis de covarianza, en los incrementos de altura, no hubo diferencias significativas entre los tratamientos de fertilización en las diferentes fechas de muestreo $(P>0,05$, datos no mostrados). En cambio, en los incrementos de diámetro si se mostraron algunas diferencias entre tratamientos, pero únicamente en las evaluaciones de agosto $(P \leq 0,01)$ y octubre del $2012(P \leq 0,03)$, siendo el tratamiento con potasio y la interacción fósforo/potasio los que presentaron estas diferencias (cuadro 3 ). No obstante, de acuerdo con la prueba de Tukey $(\alpha=0,05)$, los valores significativos mencionados anteriormente afectaron de forma negativa, pues los incrementos de diámetro fueron menores con la aplicación de potasio (T2) y fósforo/potasio (T4) en comparación de los incrementos obtenidos sin la aplicación de dichos nutrimentos (T1), los cuales fueron mayores (cuadro 4).

A pesar de la falta de diferencias estadísticas en el análisis de covarianza, se destaca un efecto positivo del tratamiento con nitrógeno (T5), puesto que en la mayoría de las evaluaciones, excepto en la de febrero 2013, hubo incrementos de diámetro superiores a los del tratamiento testigo (cuadro 4).

Variables de daño por H. grandella. La prueba de KruskalWallis mostró diferencias significativas para el número de brotes por árbol de $C$. odorata, únicamente en diciembre de 2012 con la aplicación del tratamiento de nitrógeno (cuadro 5); aunque en general, de acuerdo con la prueba Wilcoxon, estas diferencias no se manifestaron en esa variable ni en las demás variables evaluadas (número total de ataques por árbol y número de ataques por brote) (cuadro 6).

Sin embargo, a pesar de la falta de diferencias estadísticas, se manifestó un efecto positivo del potasio en la dis- 
Cuadro 3. Significancia (valor $P$ ) de los efectos de tratamientos de fertilización sobre el incremento de diámetro en árboles de $C$. odorata en las diferentes fechas de muestreo.

Significance ( $P$ value) of the effects of fertilization treatments on diameter increment of $C$. odorata in the various evaluation dates.

\begin{tabular}{|c|c|c|c|c|c|}
\hline Fuente de variación & ID08-12 & ID10-12 & ID12-12 & ID02-13 & ID04-13 \\
\hline Modelo & $0,00^{* *}$ & $0,00^{* *}$ & $0,00^{* *}$ & $0,01^{*}$ & $0,04^{*}$ \\
\hline $\mathrm{N}$ & 0,69 & 0,94 & 0,82 & 0,63 & 0,77 \\
\hline $\mathrm{P}$ & 0,06 & 0,58 & 0,44 & 0,48 & 0,66 \\
\hline K & $0,01^{*}$ & $0,03^{*}$ & 0,08 & 0,29 & 0,43 \\
\hline Interacción N/P & 0,54 & 0,80 & 0,88 & 0,49 & 0,93 \\
\hline Interacción N/K & 0,78 & 0,58 & 0.46 & 0,73 & 0,98 \\
\hline Interacción P/K & $0,01^{*}$ & 0,10 & 0,05 & 0,13 & 0,13 \\
\hline Interacción N/P/K & 0,88 & 0,95 & 0,73 & 0,51 & 0,40 \\
\hline DI & $0,00^{* *}$ & $0,00^{* *}$ & $0,00^{* *}$ & $0,00^{* *}$ & $0,00^{* *}$ \\
\hline
\end{tabular}

N: nitrógeno, P: fósforo, K: potasio. DI: diámetro inicial, ID08-12: incremento en diámetro/ agosto/ 2012, ID10-12: incremento en diámetro/ octubre/ 2012, ID12-12: incremento en diámetro/ diciembre/ 2012, ID02-13: incremento en diámetro/ febrero/ 2013, ID04-13: incremento en diámetro/ abril/ 2013. Significancia estadística de tratamientos de fertilización. *, **: Significativo a los niveles 0,05 y 0,01 de probabilidad, respectivamente. Valores sin asterisco no son significativos.

Cuadro 4. Incrementos de diámetro \pm desviación estándar en los árboles de C. odorata en cada fecha de evaluación por efecto de los tratamientos de fertilización.

Diameter and height increments \pm standard deviation of $C$. odorata trees in each date of evaluation as an effect of fertilization treatments.

\begin{tabular}{lccccc}
\hline \multirow{2}{*}{ Tratamientos } & \multicolumn{4}{c}{ Fechas de evaluación } \\
\cline { 2 - 6 } & ID08-12 & ID10-12 & ID12-12 & ID02-13 & ID04-13 \\
\hline T1= testigo (sin fertilizante) & $8,44 \pm 7,60$ & $14,86 \pm 13,98$ & $16,64 \pm 14,98$ & $17,81 \pm 17,10$ & $18,65 \pm 15,80$ \\
T2= 10 g K & $4,94 \pm 3,59$ & $10,10 \pm 7,81$ & $12,36 \pm 9,96$ & $15,07 \pm 9,96$ & $16,02 \pm 10,07$ \\
T3= 7,5 g P & $4,85 \pm 3,61$ & $11,37 \pm 8,03$ & $13,02 \pm 8,10$ & $13,91 \pm 6,81$ & $16,48 \pm 7,33$ \\
T4= 7,5 g P+10 g K & $4,93 \pm 3,61$ & $11,26 \pm 7,50$ & $13,89 \pm 8,51$ & $14,16 \pm 7,89$ & $16,32 \pm 8,97$ \\
T5= 5 g N & $8,54 \pm 5,40$ & $15,44 \pm 8,75$ & $18,78 \pm 9,94$ & $17,37 \pm 10,64$ & $19,17 \pm 10,53$ \\
T6= 5 g N+10 g P & $4,42 \pm 3,31$ & $8,80 \pm 6,19$ & $10,92 \pm 6,03$ & $11,08 \pm 9,55$ & $13,45 \pm 7,90$ \\
T7= 5 g N+7,5 g P & $5,68 \pm 4,28$ & $12,58 \pm 10,27$ & $13,79 \pm 10,79$ & $13,79 \pm 10,55$ & $14,86 \pm 10,58$ \\
T8= 5 g N+7,5 g P+10 g P & $5,56 \pm 4,92$ & $10,91 \pm 8,74$ & $13,27 \pm 9,34$ & $14,96 \pm 10,98$ & $17,37 \pm 11,35$ \\
\hline
\end{tabular}

N: nitrógeno, P: fósforo, K: potasio. DI: diámetro inicial, ID08-12: incremento en diámetro/ agosto/ 2012, ID10-12: incremento en diámetro/ octubre/ 2012, ID12-12: incremento en diámetro/ diciembre/ 2012, ID02-13: incremento en diámetro/ febrero/ 2013, ID04-13: incremento en diámetro/ abril/ 2013. Significancia estadística de tratamientos de fertilización. * **: Significativo a los niveles 0,05 y 0,01 de probabilidad, respectivamente. Valores sin asterisco no son significativos.

Cuadro 5. Significancia (valor $P$ ) para número total de brotes por árbol en C.odorata en las diferentes fechas de evaluación de acuerdo con la prueba Kruskal - Wallis. Wallis test.

Statistical significance ( $P$-value) for total number of shoots per $C$. odorata tree in the various evaluation dates, according to the Kruskal-

\begin{tabular}{lccccc}
\hline \multicolumn{1}{c}{ Factor } & NTB08-12 & NTB10-12 & NTB12-12 & NTB02-13 & NTB04-13 \\
\hline Potasio & 0,49 & 0,77 & 0,58 & 0,59 & 0,20 \\
Fósforo & 0,90 & 0,94 & 0,26 & 0,46 & 0,30 \\
Nitrógeno & 0,91 & 0,05 & $0,04^{*}$ & 0,89 & 0,39 \\
\hline
\end{tabular}

NTB08-12: número total de brotes por árbol/ agosto/ 2012, NTB10-12: número total de brotes por árbol/ octubre/ 2012, NTB12-12: número total de brotes por árbol/ diciembre/ 2012, NTB02-13: número total de brotes por árbol/ febrero/ 2013, NTB04-13: número total de brotes por árbol / abril/ 2013.*Significativo a los niveles de 0,05 de probabilidad. Valores sin asterisco no son significativos. 
Cuadro 6. Sumas de rangos para las variables de daño en C. odorata por factor experimental y fecha de evaluación de acuerdo con la prueba de Wilcoxon.

Rank sums for damage variables in C. odorata by experimental factor and evaluation date, according to the Wilcoxon test.

\begin{tabular}{|c|c|c|c|c|c|c|c|}
\hline \multirow{2}{*}{ Variables } & \multirow{2}{*}{ Evaluación } & \multicolumn{6}{|c|}{ Factor de variación } \\
\hline & & Con N & Sin $N$ & Con P & Sin $\mathrm{P}$ & Con K & Sin $\mathrm{K}$ \\
\hline \multirow{5}{*}{$\begin{array}{c}\text { Número } \\
\text { total de brotes por árbol }\end{array}$} & $08-12^{1}$ & 77,32 a & $76,68 \mathrm{a}$ & 76,62 a & 77,38 a & 74,89 a & 79,13 a \\
\hline & $10-12^{2}$ & 66,37 a & $79,37 \mathrm{a}$ & $72,76 \mathrm{a}$ & $73,76 \mathrm{a}$ & $72,04 \mathrm{a}$ & 73,96 a \\
\hline & $12-12^{3}$ & $69,20 \mathrm{a}$ & $73,60 \mathrm{a}$ & 68,38 a & 74,88 a & 69,88 a & 73,11 a \\
\hline & $02-13^{4}$ & 71,94 a & $71,06 \mathrm{a}$ & 69,15 a & 73,97 a & 73,30 a & 69,75 a \\
\hline & $04-13^{5}$ & 67,11 a & $72,76 \mathrm{a}$ & 73,39 a & 66,44 a & 74,30 a & $65,87 \mathrm{a}$ \\
\hline \multirow{5}{*}{$\begin{array}{c}\text { Número de brotes atacados por } \\
\text { árbol }\end{array}$} & $08-12^{1}$ & 79,93 а & $74,10 \mathrm{a}$ & 76,11 a & 77,86 a & 71,31 a & 82,76 a \\
\hline & $10-12^{2}$ & $72,14 \mathrm{a}$ & $73,80 \mathrm{a}$ & 74,81 a & $71,05 \mathrm{a}$ & $73,00 \mathrm{a}$ & $73,00 \mathrm{a}$ \\
\hline & $12-12^{3}$ & 72,72 a & 70,37 a & 70,31 a & 72,79 a & 70,47 a & $72,52 \mathrm{a}$ \\
\hline & $02-13^{4}$ & $71,01 \mathrm{a}$ & $71,97 \mathrm{a}$ & $72,91 \mathrm{a}$ & $70,00 \mathrm{a}$ & $70,00 \mathrm{a}$ & $72,05 \mathrm{a}$ \\
\hline & $04-13^{5}$ & $69,91 \mathrm{a}$ & 70,08 a & $70,00 \mathrm{a}$ & $69,04 \mathrm{a}$ & $72,07 \mathrm{a}$ & $68,01 \mathrm{a}$ \\
\hline \multirow{5}{*}{ Número total de ataques por árbol } & $08-12^{1}$ & 79,26 a & 74,75 a & 75,62 a & 78,42 a & 72,18 a & 81,87 a \\
\hline & $10-12^{2}$ & 72,07 a & 73,86 a & 73,86 a & 72,07 a & 71,99 a & $74,02 \mathrm{a}$ \\
\hline & $12-12^{3}$ & 72,18 a & 70,87 a & $72,25 \mathrm{a}$ & 70,81 a & $70,61 \mathrm{a}$ & $72,42 \mathrm{a}$ \\
\hline & $02-13^{4}$ & $71,51 \mathrm{a}$ & 71,48 a & 72,44 a & 70,50 a & 70,50 a & $72,47 \mathrm{a}$ \\
\hline & $04-13^{5}$ & 70,09 a & $69,90 \mathrm{a}$ & 70,90 a & 69,05 a & 72,07 a & $68,01 \mathrm{a}$ \\
\hline \multirow{5}{*}{ Número de ataques por brote } & $08-12^{1}$ & 79,89 a & 71,33 a & 74,79 a & 76,20 a & 71,97 a & $78,92 \mathrm{a}$ \\
\hline & $10-12^{2}$ & 72,50 a & 72,50 a & 72,50 a & 72,50 a & 72,50 a & $72,50 \mathrm{a}$ \\
\hline & $12-12^{3}$ & 72,19 a & $70,85 \mathrm{a}$ & 70,79 a & 72,26 a & 71,08 a & $71,91 \mathrm{a}$ \\
\hline & $02-13^{4}$ & 71,51 a & $71,48 \mathrm{a}$ & $72,44 \mathrm{a}$ & $70,50 \mathrm{a}$ & $70,00 \mathrm{a}$ & $72,47 \mathrm{a}$ \\
\hline & $04-13^{5}$ & $70,51 \mathrm{a}$ & $70,48 \mathrm{a}$ & 71,35 a & 69,59 a & $72,61 \mathrm{a}$ & $68,50 \mathrm{a}$ \\
\hline
\end{tabular}

1: evaluación agosto 2012; 2: evaluación octubre 2012; 3: evaluación diciembre 2012; 4: evaluación febrero 2013; 5: evaluación abril 2013. N: nitrógeno, P: fósforo, $\mathrm{K}$ : potasio. Letras iguales indican que no hay diferencias significativas entre tratamientos $(\alpha=0,05)$.

minución del ataque de $H$. grandella. Durante el curso de las evaluaciones, el número de brotes atacados por árbol fue disminuyendo, a excepción de abril del 2013, donde se observó mayor ataque con la presencia de potasio que sin la aplicación del nutrimento (cuadro 6). Consecuentemente, el mismo nutrimento mostró una tendencia similar en la disminución de número total de ataques por árbol (cuadro 6).

Variables fisiológicas. De acuerdo con el análisis de varianza, no hubo efectos de interacción entre los nutrimentos evaluados sobre las concentraciones de los nutrimentos analizados, únicamente hubo efectos principales altamente significativos del potasio $\left(P>0,01^{*}\right)$ sobre la concentración foliar del mismo nutrimento (cuadro 7).

No obstante, aunque sin diferencias significativas, se mostró un incremento en la concentración foliar de los nutrimentos, con tratamientos inherentes al elemento analizado (figuras 1 y 2 ).
Cuadro 7. Significancia (valor- $P$ ) según los análisis de varianza, de los efectos de los factores estudiados sobre las concentraciones de nitrógeno, fósforo y potasio en follaje de $C$. odorata.

Statistical significance ( $P$-value) according to the analyses of variance, for the effects of the studied factors on nitrogen, phosphorus and potassium concentrations in $C$. odorata foliage.

\begin{tabular}{lccc}
\hline \multirow{2}{*}{$\begin{array}{c}\text { Factor de } \\
\text { variación }\end{array}$} & \multicolumn{3}{c}{ Elemento nutritivo } \\
\cline { 2 - 4 } $\mathrm{N}$ & Nitrógeno (N) & Fósforo (P) & Potasio (K) \\
$\mathrm{P}$ & 0,60 & 0,88 & 0,80 \\
$\mathrm{~K}$ & 0,60 & 0,85 & 0,92 \\
$\mathrm{NxP}$ & 0,37 & 0,97 & $0,01^{*}$ \\
$\mathrm{NxK}$ & 0,30 & 0,60 & 0,89 \\
PxK & 0,70 & 0,95 & 0,75 \\
NxPxK & 0,88 & 0,64 & 0,37 \\
\hline
\end{tabular}

$\mathrm{N}$ : nitrógeno, $\mathrm{P}$ : fósforo, $\mathrm{K}$ : potasio. ${ }^{*} P \leq 0,01$. Valores sin asterisco no son significativos. 


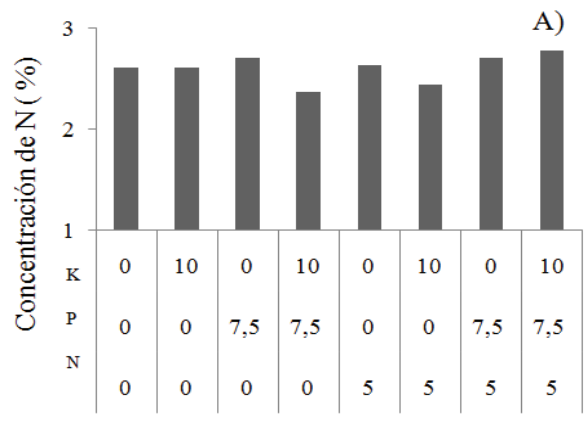

Tratamientos $(\mathrm{g})$

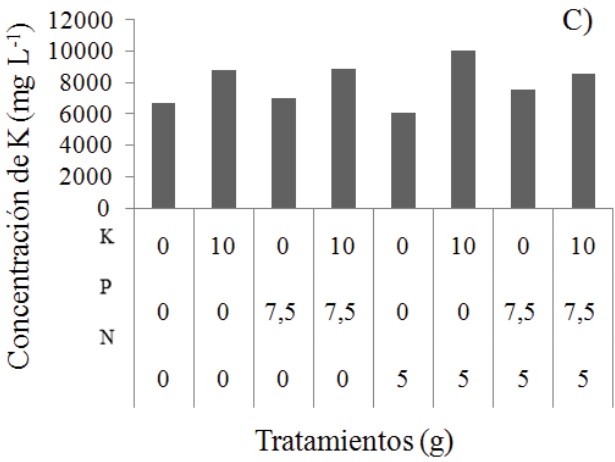

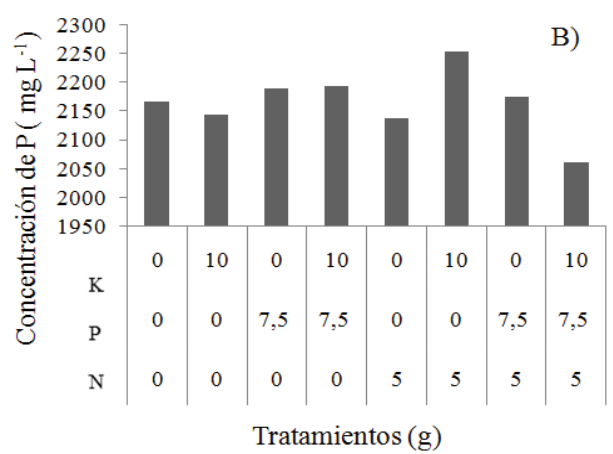

Combinación de los tratamientos

T1: Testigo (sin nutrimento).

T2: Potasio (K).

T3: Fósforo (P).

T4: Fósforo, potasio $(\mathrm{P} * \mathrm{~K})$.

T5: Nitrógeno $(\mathrm{N})$.

T6: Nitrógeno, potasio $\left(\mathrm{N}^{*} \mathrm{~K}\right)$.

T7: Nitrógeno, fósforo (N*P).

T8: Nitrógeno, fósforo, potasio (N*P*K).

Figura 1. Comportamiento del crecimiento, concentraciones y contenidos de A) nitrógeno, B) fósforo y C) potasio en follaje de C. odorata en respuesta a tratamientos de fertilización.

Effect of fertilization treatments on foliage concentrations of A) nitrogen, B) phosphorus and C) potassium in C. odorata.
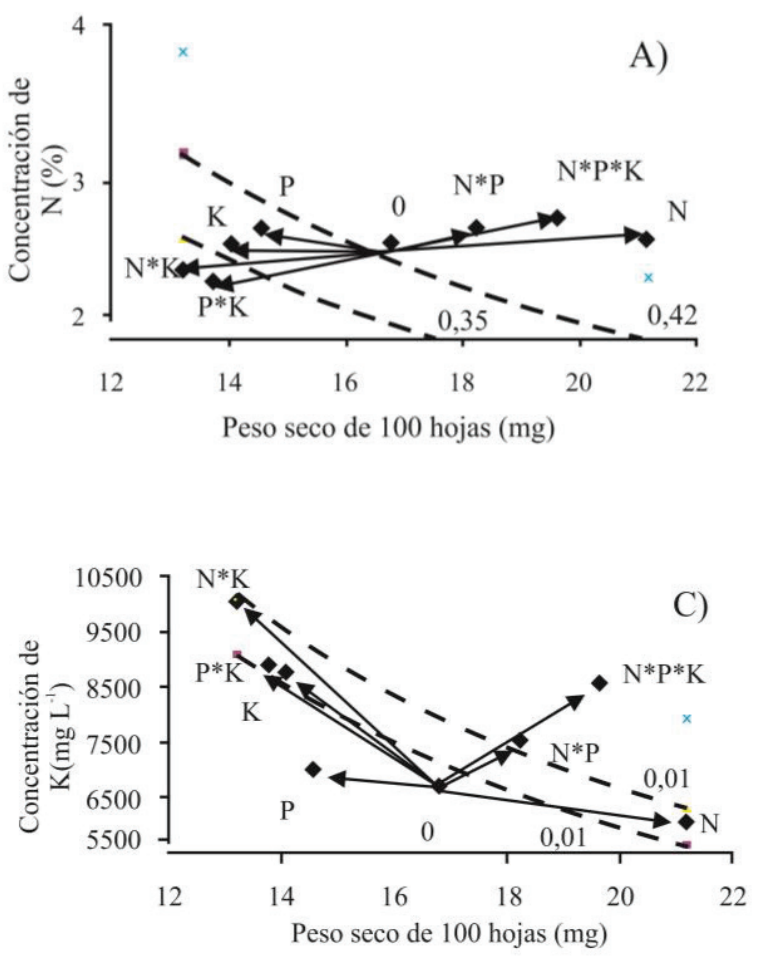

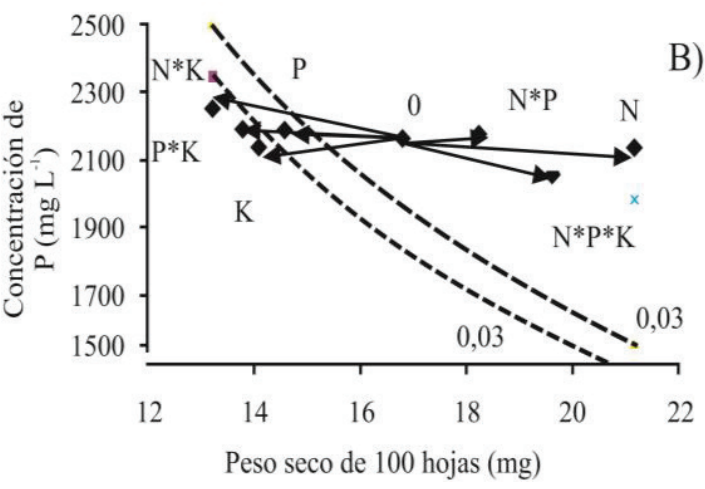

Combinación de los tratamientos
T1: Testigo (sin nutrimento).
T2: Potasio (K).
T3: Fósforo $(\mathrm{P})$.
T4: Fósforo, potasio $(\mathrm{P} * \mathrm{~K})$.
T5: Nitrógeno $(\mathrm{N})$.
T6: Nitrógeno, potasio $\left(\mathrm{N}^{*} \mathrm{~K}\right)$.
T7: Nitrógeno, fósforo $(\mathrm{N} * \mathrm{P})$.
T8: Nitrógeno, fósforo, potasio $\left(\mathrm{N}^{*} \mathrm{P} * \mathrm{~K}\right)$.

Figura 2. Efecto de los tratamientos de fertilización sobre las concentraciones foliares de A) nitrógeno, B) fósforo y C) potasio en C. odorata.

Behavior of growth, concentrations and contents of A) nitrogen, B) phosphorus and C) potassium in foliage of C. odorata in response to fertilization treatments. 


\section{DISCUSIÓN}

Nutrimentos individuales aplicados y sus interacciones sobre el crecimiento de C. odorata. Con base en los resultados obtenidos, el crecimiento en altura no presenta diferencias estadísticas asociadas a los tratamientos de fertilización debido a que esta variable fue alterada anormalmente por las decapitaciones constantes que el barrenador provocó en las yemas apicales de los árboles. En trabajos futuros se recomienda considerar este aspecto. En cambio en el crecimiento en diámetro, aunque en general la falta de diferencias estadísticas en la mayoría de las evaluaciones (cuadro 3), evidencie la ausencia de efectos positivos de la fertilización, es notable la función del nitrógeno sobre la promoción del crecimiento de la especie. Tanto en sistemas forestales naturales (Pasquini y Santiago 2012) como en plantaciones (Álvarez et al. 1999) tal efecto se ha manifestado, principalmente por el rol que el nitrógeno tiene sobre la actividad fotosintética de los árboles. Probablemente no se tiene un efecto sobresaliente del nitrógeno (cuadro 4) porque la dosis (5 g de urea por planta en cada aplicación) resulta baja ante las demandas o condiciones de la plantación; primero, porque hay deficiencia de nitrógeno en el sitio de plantación, esto con base en lo reportado por Drechsel y Zech (1994) y en las características del suelo del sitio; segundo, debido al ineficiente control de malezas, pues estas eran abundantes y podrían haber competido por el recurso mineral; tercero, por el mal drenaje del sitio, ya que la textura (cuadro 1) y la orografía (bajío con poca pendiente) genera inundaciones constantes que pudieran estar limitando la disponibilidad de oxígeno y, por ende, la disponibilidad del nutrimento (Kreuzwieser y Gessler 2010); cuarto, debido a pérdidas por volatilización, pues el tipo de fuente nitrogenada (urea) es particularmente susceptible a volatilización cuando la temperatura y el pH es alto (Haynes y Sherlock 1986). Estas condiciones estuvieron presentes en el sitio al momento de realizar la primera aplicación de fertilizante. Sin embargo, ante toda esa gama de posibilidades, podría ser que el factor principal que limita las respuestas del nitrógeno es el disturbio ocasionado por la plaga. Ante el ataque de la plaga, las plantas podrían estar generando reservas de defensa o destinando recursos para compensar la pérdida de biomasa, lo cual directamente compite con el crecimiento (Chapin et al. 1990), además grandes cantidades de recursos podrían estarse perdiendo en la biomasa removida, limitando la cantidad de recursos disponibles para la reubicación, proceso por el cual, según Smethurst (2010), los árboles satisfacen sus necesidades inmediatas por recursos nutrimentales.

Por otro lado, aunque no se tuvo una respuesta similar a la del nitrógeno, la falta de efectos positivos con la aplicación de fósforo, a pesar de la deficiencia de este nutrimento en el sitio (Hart et al. 2004) (cuadro 2), se atribuye al efecto que este nutrimento tiene sobre la disponibilidad y absorción del zinc, cuya interacción negativa con el fósforo es ampliamente conocida (Mengel y Kirkby 1982,
Fernández 2007). Al darse este mecanismo, se genera una deficiencia de zinc en la planta con la consecuente disminución del crecimiento, y dado a que en el sitio el zinc es deficiente, tal repuesta se incrementa (Pravin et al. 2013) (cuadro 2). Adicionalmente, también algunos de los factores discutidos con el nitrógeno, podrían estar implicados, principalmente al que refiere al disturbio ocasionado por la plaga.

Para el potasio también se asume hipotéticamente que el efecto colateral de la plaga es el principal responsable de la falta de respuestas favorables en crecimiento. Sin embargo, en primera instancia, la falta de humedad en el suelo cuando se hizo la fertilización podría haber reducido la ruta de difusión del potasio hacia las raíces, factor que según Hawkesford et al. (2012), es uno de los principales que determina la disponibilidad del nutrimento.

Finalmente, para el caso de las interacciones entre los nutrimentos, se considera que la ausencia de respuestas tal y como se presentaron en los casos anteriores, se debe a la complementariedad que tienen estos macronutrimentos sobre el metabolismo de las plantas (Hawkesford et al. 2012).

Variables de daño de H. grandella. Los efectos que se presentaron en el crecimiento por los tratamientos de fertilización, reflejan que con las dosis aplicadas de los nutrimentos ya sea de manera individual o combinada, no se mejora el balance nutrimental de los árboles y, por ende, no se favorecen mecanismos de defensa que permitan a las plantas una reducción considerable en la incidencia de la plaga. De haber obtenido respuestas positivas en el balance nutrimental, podría haberse tenido el efecto esperado sobre la plaga, como lo obtuvo López y Estañol (2007), quienes tras detectar y corregir deficiencia nutrimentales en árboles de Pinus leiophylla Schlecht et Cham demuestran que este aspecto es crucial para reducir el ataque de plagas, principalmente porque la cantidad de nutrientes disponibles es un factor que determina el grado de susceptibilidad de las plantas ante este tipo de agentes (Pérez et al. 2013).

La tendencia mostrada por el potasio en la reducción del número de ataques (cuadro 6), se atribuye al efecto que tiene este mineral sobre la reducción del ataque de plagas y enfermedades (Hawkesford et al. 2012).

Variables fisiológicas. En general, las respuestas en crecimiento e incidencia de la plaga discutidas previamente y las obtenidas con los análisis químicos y los nomogramas de vectores, reafirman que con los tratamientos implicados en general no se mejora el estatus nutrimental de los árboles y por ello no se tienen las respuestas esperadas, pues no se favorece un consumo de lujo que permita la formación de reservas de defensa o recuperación sin comprometer el crecimiento. Los nomogramas (figura 2A), reflejan para el caso del nitrógeno, el efecto que de alguna manera tuvo sobre el crecimiento, pero también muestra las deficiencias en el sitio (cuadro 1) y la dilución provocado por la siner- 
gia entre ambos factores. Con relación al fósforo (figura 2B), se muestra una concentración la cual fue dada por la posible interacción con el zinc que no favorece el crecimiento. Finalmente, para el potasio (figura 2C), los nomogramas contradicen la hipótesis planteada sobre el efecto negativo de la sequía, puesto que se aprecia una concentración elevada del nutrimento en las hojas, lo que indica que si fue absorbido por los árboles aunque este no favoreció el crecimiento. Para este caso y el de fósforo, procesos metabólicos de almacenamiento de nutrientes principalmente de acumulación podría haber estado implicados. Chapin et al. (1990) reconocen tres tipos de almacenamiento de nutrientes en las plantas: acumulación, formación de reservas y reciclaje. Los nutrientes que se acumulan son aquellos que se almacenan como compuestos inorgánicos, y los que forman reservas son aquellos que se almacenan como compuestos orgánicos. El fósforo y el potasio se relacionan con el primer caso, mientas que el nitrógeno con el segundo. Finalmente, esto también explica la razón de porque hubo mayor concentración fósforo y potasio en el follaje que de nitrógeno. Respuestas similares sobre este aspecto son reportadas por Pasquini y Santiago (2012).

\section{CONCLUSIONES}

En estudios similares, debido al patrón de ataque de Hypsipyla grandella, la altura no es una variable confiable para evaluar el crecimiento de C. odorata. El crecimiento en diámetro se favorece con la aplicación de dos dosis de $5 \mathrm{~g}$ urea, pero su eficiencia es limitada ante la interacción entre factores edafológicos del sitio y el ataque de la plaga. La fertilización con $10 \mathrm{~g}$ de sulfato de potasio muestra un efecto positivo en la reducción del ataque de la plaga, aunque no contundente. En general los nutrimentos mediante las dosis aplicadas no manifiestan una mejora del estatus nutrimental de las plantas que implique una reducción en la incidencia del barrenador. Se recomienda que en trabajos posteriores con similar enfoque se prueben otras dosis o una mayor gama de nutrimentos.

\section{REFERENCIAS}

Álvarez MJ, J Rodríguez, D Suárez F. 1999. Mejoramiento de la productividad de Pinus radiata D. Don, a través de un método racional de fertilización. Bosque 20(1): 23-36.

Barboza J, L Hilje, J Durón, V Cartín, M Calvo. 2010. Fagodisuasión de un extracto de ruda (Ruta chalepensis, Rutaceae) y sus particiones sobre larvas de Hypsipyla grandella (Lepidoptera: Pyralidae). Revista de Biología Tropical 58: 01-14.

Briceño AJ. 1997. Aproximación hacia un manejo integrado del barrenador de las meliáceas, Hypsipyla grandella Zeller. Revista Forestal Venezolana 4 (1): 27-23.

Chapin FSIII, ED Schulze, HA Mooney. 1990. The ecology and economics of storage in plants. Annual Review of Ecology and Systematics 21: 423-447.

Cibrián TD, JT Méndez, R Campos, HO Yates, J Flores. 1995.
Insectos forestales de México. México DF, México. Universidad Autónoma Chapingo. p. 112-115.

Drechsel P, y W Zech.1994. DRIS evaluation of teak (Tectona grandis L. f) mineral nutrition and effects of nutrition and site quality on teak growth in West Africa. Forest Ecology and Management 70: 121-133.

Fernández MT. 2007. Fósforo: amigo o enemigo. ICIDCA. Sobre los derivados de la Caña de Azúcar 41(2): 51-57.

Goulet E, A Rueda, A Shelton. 2005. Management of the mahogany shoot borer, Hypsipyla grandella (Zeller) (Lepidoptera:Pyralidae), through weed management and insecticidal sprays in 1- and 2-year-old Swietenia humilis Zucc. plantations. Crop Protection 24: 821-828.

Hart J, R Fletchert, C Landgred, D Horneck, S Webster, M Bondi. 2004. Christmas tree nutrient management guide for Western Oregon and Washington. Corvallis, OR, USA. Oregon State University. 60 p.

Haynes RJ, RR Sherlock. 1986. Gaseous losses of nitrogen. In Haynes RJ ed. Mineral nitrogen in the plant-soil system. London, United Kingdom. Academic Press. p. 242-285.

Hawkesford M, W Horst, T Kichey, H Lambers, J Schjoerring, IS Møller, P White. 2012. Functions of macronutrients. In Marschner P ed. Mineral nutrition of higher plants. Third edition. Oxford, UK. Elsevier. p. 135-189.

Julian P, SD Eigenbrode, L Hilje, RR Tripepi, ME Aguilar, F Mesen. 2010. Use of grafting to prevent Hypsipyla grandella Zeller (Lepidoptera:Pyralidae) damage to new world meliaceae species. Neotropical Entomology 39: 618-625.

Kreuzwieser J, A Gessler. 2010. Global climate change and tree nutrition: influence of water availability. Tree Physiology 30: 1221-1234.

Lim GT, LG Kirton, SM Salom, LT Kok, RD Fell, DG Pfeiffer. 2008. Mahogany shoot borer control in Malaysla and prospects for biocontrol using weaver ants. Journal of Tropical Forest Science 20: 147-155.

López MA, E Estañol. 2007. Detección de deficiencias de hierro en Pinus leiophylla a partir de los efectos de dilución y concentración nutrimental. Terra Latinoamericana 25(1): 9-15.

Mancebo F, Luko Hilje, Gerardo A. Mora, Salazard R. 2002. Biological activity of two neem (Azadirachta indica A. Juss., Meliaceae) products on Hypsipyla grandella (Lepidoptera: Pyralidae) larvae. Crop Protection 21: 107-112.

Mengel K, AE Kirkby. 1982. Principles of plant nutrition. International Potash Institute. Berna. 593p.

Mery G, S Kegen, C Luján. 2001. Forest - Based Development in Brazil, Chile y México. In Palo M, J Uusivouri, G Mery eds. Londres, UK. Kluwer Academic Publishers. p. 243-263.

Mengel K, AE Kirkby. 1982. Principles of plant nutrition. Berna, Suiza. International Potash Institute. 593 p.

Opuni-Frimpong E, DF Karnosky, AJ Storer, JR Cobbinah. 2008. Silvicultural systems for plantation mahogany in Africa: Influences of canopy shade on tree growth and pest damage. Forest Ecology and Management 255: 328-333.

Pasquini SC, LS Santiago. 2012. Nutrients limits photosynthesis in seedlings of a lowland tropical forest tree species. Oecologia 168(2): 311-319. DOI: 10.1007/s00442-011-2099-5

Pérez CM, MA López, A Equihua, VM Cetina, JT Méndez. 2013. Relationships between site factor and bark beetle attack on pine trees. Journal of Biological Sciences 13(7): 621-627.

Pérez-Salicrup DR, R Esquivel. 2008. Tree infection by Hypsi- 
pyla grandella in Swietenia macrophylla and Cedrela odorata (Meliaceae) in Mexico's southern Yucatan Peninsula. Forest Ecology and Management 255: 324-327.

Pennington TD, J Sarukhán. 2005. Árboles tropicales de México. Manual para la identificación de las principales especies. Tercera edición. México DF, México. Ediciones Científicas Universitarias. 511 p.

Pravin RC, DV Ahire, VD Ahire, M Chkravarty, S Maity. 2013. Soil bulk density as related to soil texture, organic matter content and available total nutrients of Coimbatore soil. International Journal of Scientific and Research Publications 3(2): 1-8.

Silva ML, J Cardoso, AC Maciel, O Shigueo, V Melo, G Rodrigues, AJ Silva, IJ Matos, EC Medeiros. 2014. Crescimento de mogno-brasileiro e resistência a Hypsipyla grandella em função do cálcio e do boro. Revista Árvore 38(6): 1085-1094.

Smethurst PJ. 2010. Forest fertilization: trends in knowledge and practice compared to agriculture. Plant and Soil 335: 83-100.

Recibido: 16.06 .14

Aceptado: 25.03.15 
